\title{
Dynamics of plc gene transcription and $\alpha$-toxin production during growth of Clostridium perfringens strains with contrasting $\alpha$-toxin production ${ }^{\text {th }}$
}

\author{
Lone Abildgaard ${ }^{\mathrm{a}, \mathrm{b}, \mathrm{c}}$, Andreas Schramm ${ }^{\mathrm{b}}$, Knut Rudi ${ }^{\mathrm{c}, \mathrm{d}}$, Ole Højberg ${ }^{\mathrm{a}, *}$ \\ ${ }^{a}$ Department of Animal Health and Bioscience, Faculty of Agricultural Sciences, Aarhus University, P.O. Box 50, DK-8830 Tjele, Denmark \\ ${ }^{\mathrm{b}}$ Department of Biological Sciences, Microbiology, Aarhus University, Ny Munkegade, Building 1540, DK-8000 Aarhus C, Denmark \\ ${ }^{\mathrm{c}}$ Nofima Mat, Norwegian Food Research Institute, Osloveien 1, N-1430 Ås, Norway \\ ${ }^{\mathrm{d}}$ Hedmark University College, Department of Natural Sciences, N-2318 Hamar, Norway
}

\section{A R T I C L E I N F O}

\section{Article history:}

Received 17 December 2008

Received in revised form 29 April 2009

Accepted 28 May 2009

\section{Keywords:}

C. perfringens

$\alpha$-Toxin production

plc transcription

Real-time PCR

\begin{abstract}
A B S T R A C T
The aim of the present study was to investigate transcription dynamics of the $\alpha$-toxinencoding plc gene relative to two housekeeping genes ( $g y r A$ and $r p l L$ ) in batch cultures of three Clostridium perfringens strains with low, intermediate, and high levels of $\alpha$-toxin production, respectively. The plc transcript level was always low in the low $\alpha$-toxin producing strain. For the two other strains, plc transcription showed an inducible pattern and reached a maximum level in the late exponential growth phase. The transcription levels were however inversely correlated to $\alpha$-toxin production for the two strains. We propose that this discrepancy is due to differences in plc translation rates between the strains and that strain-specific translational rates therefore must be determined before $\alpha$ toxin production can be extrapolated from transcript levels in $C$. perfringens.
\end{abstract}

(c) 2009 Elsevier B.V. All rights reserved.

\section{Introduction}

Necrotic enteritis (NE) is a common disease in broiler chickens caused predominantly by Clostridium perfringens (Songer, 1996). Usually, NE occurs 2-6 weeks after hatching and is characterized by the sudden onset of diarrhea and mucosal necrosis caused by overgrowth of $C$. perfringens in the small intestine (Ficken and Wages, 1997; Fukata et al., 1991). The mere presence of $C$. perfringens does not necessarily lead to disease but at a critical population size, toxin production results in necrotization of the gastrointestinal epithelium from random foci (Cowen et al., 1987; Engberg et al., 2002; Ficken and Wages, 1997; Pedersen et al., 2003). The mechanisms behind establishment of infection are not fully determined and several new virulence

\footnotetext{
Nucleotide sequence data reported are available in the GenBank databases under the accession numbers EU834132-EU834179.

Abbreviations: NE, necrotic enteritis; RT, reverse transcription; MDAP, maximum detected $\alpha$-toxin production.

* Corresponding author. Tel.: +45 89991183; fax: +45 89991166.

E-mail address: ole.hojberg@djf.au.dk (O. Højberg).
}

factors and mechanisms have been suggested recently (Keyburn et al., 2008; Kulkarni et al., 2008; Olkowski et al., 2008). The $\alpha$-toxin (phospholipase $C$ ) is however believed to play an important role in the severity of the disease (AlSheikhly and Truscott, 1977a, 1977b; Awad et al., 1995; Fukata et al., 1988; Thompson et al., 2006; Williamson and Titball, 1993) and immunization with recombinant $\alpha$-toxin has been shown partly to protect broilers in experimental challenges with C. perfringens (Cooper et al., 2009; Kulkarni et al., 2007). The dynamics of $\alpha$-toxin production during growth of different $C$. perfringens strains are poorly understood. The goal of this study was therefore to investigate the relationship between growth phase, plc gene transcription, and $\alpha$-toxin production in three $C$. perfringens strains representing low, intermediate and high levels of $\alpha$-toxin production, respectively.

\section{Materials and methods}

\subsection{Bacterial strains and growth conditions}

In previous studies, 60 strains of $C$. perfringens with different pulsed-field gel electrophoresis profiles were 
isolated from chickens, analyzed for their $\alpha$-toxin sequence type (Abildgaard et al., 2009; Pedersen et al., 2003) and 35 isolates were further screened for production of $\alpha$-toxin in batch cultures (Abildgaard et al., 2009) similar to the approach used by Gholamiandekhordi et al. (2006). Based on this screening, the following three strains were selected for more detailed studies: (i) strain T2 (sequence type: VIe (Abildgaard et al., 2009); source: NE chicken; $\alpha$-toxin production level: low, $<1 \%$ of maximum detected $\alpha$-toxin production (MDAP) of the 35 strains); (ii) strain S3 (sequence type: $\mathrm{Vb}$; source: healthy chicken; $\alpha$ toxin production level: intermediate, $57 \%$ of MDAP of the 35 strains); and (iii) strain T9 (sequence type: Vb; source: NE chicken; $\alpha$-toxin production level: high, $85 \%$ of MDAP of the 35 strains). The strains were maintained in Anaerobe Basal Broth (CM0957; Oxoid Ltd., Basingstoke, Hampshire, UK) at $30{ }^{\circ} \mathrm{C}$; $500-\mathrm{ml}$ infusion bottles with $400 \mathrm{ml}$ broth were inoculated $(0.1 \%)$ from overnight cultures, flushed $\left(80 \% \mathrm{~N}_{2}, 10 \% \mathrm{CO}_{2}\right.$, and $\left.10 \% \mathrm{H}_{2}\right)$ and incubated at $37{ }^{\circ} \mathrm{C}$ under agitation $(120 \mathrm{rpm})$ to avoid aggregation and subcultures in different growth phases. At regular intervals, growth was measured as $\mathrm{OD}_{600}$, and samples were withdrawn for quantification of plc mRNA by reverse transcriptase quantitative PCR (RT-qPCR; see below) and of $\alpha$-toxin by the ELISA method previously described (Abildgaard et al., 2009). All experiments were performed in triplicates. The three selected strains were further analyzed for their $\alpha$-toxin production pattern ( $\alpha$-toxin accumulation in overnight batch cultures) to check for potential inter-strain differences in intracellular pools and/ or differences in protease degradation of the $\alpha$-toxin. For this purpose, batch cultures of the strains were inoculated $(0.1 \%)$ from pre-cultures as outlined above and grown overnight (approximately $16 \mathrm{~h}$ ) at $37^{\circ} \mathrm{C}$ in $10 \mathrm{ml}$ anaerobe basal broth. The suspensions were centrifuged and the supernatants and the pellets were immediately frozen separately in liquid nitrogen and stored at $-80^{\circ} \mathrm{C}$. The frozen pellets were re-suspended in $1 \mathrm{ml}$ TES buffer (define) and $500 \mu \mathrm{l}$ was transferred to Eppendorf tubes containing $200 \mathrm{mg}$ glass beads $(0.18 \mathrm{~mm})$ and amended with $5 \mu \mathrm{l} 100 \times$ Protease Inhibitor Cocktail Set I (539131, CALBIOCHEM $^{\circledR}$, San Diego, CA). The suspensions were Vortexed ( $1 \mathrm{~min}$ ), $5 \mu$ l lysozyme $\left(10 \mathrm{mg} \mathrm{ml}^{-1}\right)$ added and the tubes were incubated $30 \mathrm{~min}$ at $37^{\circ} \mathrm{C}$. The tubes were Vortexed ( $1 \mathrm{~min}$ ) again, centrifuged and the supernatants were frozen in liquid nitrogen and stored at $-80^{\circ} \mathrm{C}$. The supernatants from the overnight cultures as well as from the lysed cells were analyzed for $\alpha$-toxin content by the previously described ELISA method (Abildgaard et al., 2009), where the former were amended with the protease inhibitor as well prior to the analysis.

\subsection{TaqMan probes and primers}

Published primers and probes for qPCR (Skånseng et al., 2006) showed several mismatches to the recently extended plc gene sequence set (Abildgaard et al., 2009). To exclude target underestimation caused by destabilizing mismatches (Werbrouck et al., 2007), a new primer/probe set (Table 1) was designed using the Primer Express software package (v. 2.0; Applied Biosystems, Stockholm,
Table 1

Primers (F and R) and probes (T) for RT-qPCR.

\begin{tabular}{lll}
\hline Target & Name & Sequence $\left(5^{\prime}-3^{\prime}\right)$ \\
\hline Plc & $398 \mathrm{~F}$ & CTA GAT ATG AAT GGC AAA GAG GAA ACT A \\
& $475 \mathrm{R}$ & AAC ATT GCA GGA TGA TAT GGA GTA GTA TCT AT \\
& 430T & CAA GCT ACA TTC TAT CTT GGA GAG GCT \\
& & ATG CAC TAT TT \\
RplL & 3F & GAC AAA AGA GCA AAT CAT AGA AGC TAT AA \\
& 125R & TCC GCC TAC AAC AGC AAC TG \\
& 69T & TGT GAA GAA GAA TTC GGA TTC GGA GTA \\
& & AGC GCT GC \\
GyrA & 693F & CAT TAA AGG ACC AGA TTT CCC TAC AG \\
& 771R & TTT TCC AGT TTC ATA AGC AGC TCT T \\
& 720T & AGG AAT AAT AAT GGG TAA ATC AGG AA
\end{tabular}

Sweden), and checked for potential cross-reactivity against the GenBank database (http://www.ncbi.nlm.nih.gov). Positions 339-340 were avoided as target sites, since an intron between these positions had been observed in strain S3 and other strains of $C$. perfringens (Abildgaard et al., 2009; Ma et al., 2007). Two constitutively expressed genes were selected for normalization of plc transcript levels: gyrA, encoding DNA gyrase (Huang, 1996), and $r p l L$, encoding the ribosomal protein L7/L12 (Ramakrishnan and Moore, 2001). Sequences for gyrA and $r p l L$ were retrieved from $24 \mathrm{C}$. perfringens strains (GenBank accession numbers: EU834132-EU834179) isolated from chickens using published primers (Rooney et al., 2006), and were used, together with 124 gyrA and $244 \mathrm{rplL}$ sequences of $C$. perfringens isolated from various sources (Rooney et al., 2006), for probe and primer design (Table 1 ).

\subsection{Quantitative real-time $R T-P C R$ amplification}

Samples of RNA from strains S3, T2, and T9 were preserved by transferring aliquots of $0.5 \mathrm{ml}$ culture to $2 \mathrm{ml}$ microcentrifuge tubes containing $1 \mathrm{ml}$ RNA protect (QIAGEN). Total RNA was isolated using the RNAprotect protocol (QIAGEN) which includes enzymatic lysis, proteinase $\mathrm{K}$ digestion, and mechanical disruption, and purified using the RNeasy mini kit (QIAGEN) according to the manufacturer's recommendations. The RNA was eluted twice in $20 \mu$ l RNase free water. The QIAGEN RNaseFree DNase Set was applied during RNA extraction to remove DNA. The concentration and purity of the RNA was determined as absorbance at 260 and $280 \mathrm{~nm}$ in a NanoDrop ND-1000 Spectrophotometer (Thermo Fisher Scientific, Wilmington, DE, USA). The RNA was stored at $-80{ }^{\circ} \mathrm{C}$ for a maximum of 1 week before cDNA synthesis to minimize differential RNA degradation reported for longer storage (Alifano et al., 1994; Rudi et al., 2003). Reverse transcription (RT) was performed using the first-strand cDNA protocol and SuperScriptTMIII reverse transcriptase (Invitrogen, Carlsbad, CA, USA) according to the manufacturer's recommended protocol with $100 \mathrm{ng}$ RNA and $100 \mathrm{ng}$ random hexamer primers (Invitrogen) per reaction. The thermal profile was as follows: $25^{\circ} \mathrm{C}$ for $5 \mathrm{~min}, 50^{\circ} \mathrm{C}$ for $60 \mathrm{~min}$, and $70{ }^{\circ} \mathrm{C}$ for $15 \mathrm{~min}$. The resulting cDNA ( $1 \mu \mathrm{l}$ in a total of $10 \mu \mathrm{l}$ reaction volume) was amplified with TaqMan Universal PCR Master Mix (Applied Biosystems, Stockholm, Sweden) with $0.4 \mathrm{mg} \mathrm{ml}^{-1}$ bovine serum 
albumin (Fermentas, Helsingborg, Sweden) and $0.5 \mathrm{pmol}$ $\mu l^{-1}$ primers and $0.1 \mathrm{pmol}_{\mu l^{-1}}$ probe (Table 1 ). The probes contained 6-carboxyfluorescein (FAM) at the $5^{\prime}$ end and either 6-carboxytetramethylrhodamine (TAMRA) (plc and $r p l L)$ or a non-fluorescent quencher with the MGB protein (Applied Biosystems) at the $3^{\prime}$ end (gyrA). A 7900HT sequence detection system (Applied Biosystems) was used for $\mathrm{qPCR}$ with the following temperature settings: 2 min start up at $50{ }^{\circ} \mathrm{C}, 10 \mathrm{~min}$ initial denaturation at $95^{\circ} \mathrm{C}$, and 40 cycles of denaturing at $95^{\circ} \mathrm{C}(15 \mathrm{~s})$ and annealing/extension at $60{ }^{\circ} \mathrm{C}(1 \mathrm{~min})$. Data were analyzed with the SDS 2.1 application software (Applied Biosystems). A standard curve $\left(7 \times 10^{5}\right.$ to 70 genomes $\left.\mu l^{-1}\right)$ was prepared by serial dilution of $C$. perfringens genomic DNA of known concentration, using the genome size of $C$. perfringens strain 13 and Avogadro's number for calculations. Since plc, gyrA, and rplL are single copy genes in $C$. perfringens (Shimizu et al., 2002), cycle thresholds $\left(C_{\mathrm{T}}\right.$ values) were directly converted to gene copy numbers for the standards; results are expressed as number of plc transcripts relative to gyrA or $r p l L$ transcript numbers.

\section{Results}

\subsection{Levels of $\alpha$-toxin production}

The relative levels of extra cellular $\alpha$-toxin accumulation in batch cultures of the three strains (T9: high; S3: intermediate; and T2: low/insignificant) as illustrated in Fig. 1D were highly reproducible and could be repeated with months interval (data not shown). In a control experiment it was further observed that the intracellular pools of $\alpha$-toxin were insignificant. Thus, $\alpha$-toxin could only be detected in lysate from the high producing strain T9 and at a concentration $500 \times$ lower than in the supernatant. The samples, taken from the control experiments, were all amended with protease inhibitor during the analysis to avoid any bias caused by differences in $\alpha$ toxin degradation between the strains.

\subsection{Design of RT-real-time PCR protocol}

While all primers had a perfect match to their target site, the large gyrA sequence variation caused problems for designing a suitable probe: despite the design as minor groove-binding (MGB) probe, which forms an extremely stable duplex with single-stranded DNA and therefore allows shorter probe lengths (Kutyavin et al., 2000), one mismatch remained to gyrA of strain T2; plc transcript levels normalized against gyrA might therefore be slightly overestimated for T2 (Fig. 1B). The log-linear range for all qPCR reactions spanned five orders of magnitude with an $r^{2}>0.99$ (Fig. S1). The regression curves for $p l c$, gyrA, and rplL had slopes of $3.33,3.37$, and 3.32 , corresponding to amplification efficiencies of $0.99,0.98$, and 1.00 , respectively. The contribution of genomic DNA to the CDNA signal was assessed by determination of the $\Delta C_{\mathrm{T}}$ for the RT-qPCR with and without addition of reverse transcriptase. During exponential growth, all samples tested had a $\Delta C_{\mathrm{T}}>4$, indicating a contribution of genomic DNA of $<10 \%$ to the cDNA signal (calculated from the slope of the regression

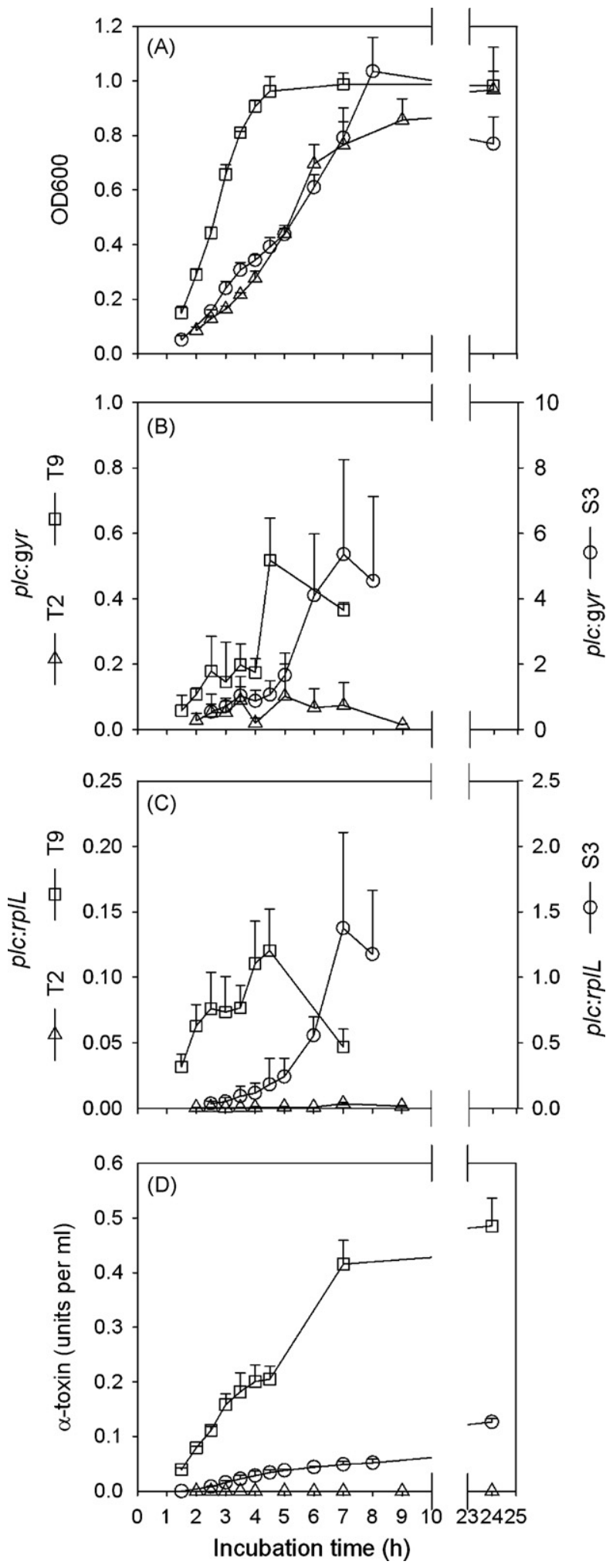

Fig. 1. Growth measured as $\mathrm{OD}_{600}(\mathrm{~A})$, transcription of plc relative to gyrA (B) and $r p l L(C)$, and $\alpha$-toxin production (D) of C. perfringens strain S3 (O), T9 $(\square)$, and T2 $(\triangle)$. Error bars, standard deviations $(n=3)$. Error bars smaller than symbols are not shown. 
line). This value is within experimental variation and does not interfere with the transcript quantification. In contrast, the $\Delta C_{\mathrm{T}}$ was $<0.5$ in late stationary phase, resulting in transcript levels below the detection limit of the assay.

\subsubsection{Transcript levels of plc and production of $\alpha$-toxin during growth of $C$. perfringens}

The growth of the three different strains reached the early exponential phase and early stationary phase after $1-$ 2 and 5-9 h, respectively (Fig. 1A).

The plc transcription dynamics showed the same trend irrespective which transcripts (gyrA or $r p l L$ ) were used for normalization (Fig. 1B and C). Transcript levels of plc strongly differed between the three strains; for strain T2, it was just around the detection limit throughout the entire growth phase, and no toxin was detected in the culture supernatant, although low levels of $\alpha$-toxin production had been observed for this strain in an earlier study (Abildgaard et al., 2009). Strains T9 and S3 showed much higher plc transcript levels and a 4- to 30-fold increase from early to late exponential phase (Fig. 1B and C). Although following the same dynamics, the relative transcript level of strain S3 was however up to 10 times higher than the level observed for strain T9 (Fig. 1B and C).

\section{Discussion}

\subsection{Levels of $\alpha$-toxin production}

The reproducibility of the levels of $\alpha$-toxin accumulation measured in supernatants of the batch cultures combined with the fact that only insignificant levels of the toxin were detectable in cell lysates, support the use of secreted $\alpha$-toxin as a measure of the strain-specific production pattern in accordance with previously reported observations (Abildgaard et al., 2009; Gholamiandekhordi et al., 2006). It is however important to emphasize that comparison should only be made between strains grown under comparable conditions, since, e.g., culture media and $\mathrm{pH}$ have been reported to influence toxin production levels of $C$. perfringens significantly (Fernandez-Miyakawa et al., 2007; Möllby et al., 1976).

\subsubsection{Transcript levels of plc and production of $\alpha$-toxin during growth of $C$. perfringens}

Comparison of Fig. 1A-C indicates that transcription of plc is induced in exponential phase rather than being constitutive. Peaking of $\alpha$-toxin accumulation in exponential phase has previously been reported (Bullifent et al., 1996), but to our knowledge, the present study is the first to demonstrate induced plc transcription in direct comparison to housekeeping genes. The expression pattern of plc could potentially reflect an evolutionary strategy to increase substrate availability in crowded environments by increasing toxin (phospholipase) production. A similar up-shift in transcription of the gene encoding botulinum neurotoxin (cntA) has been observed in Clostridium botulinum (Sharkey et al., 2005; Shin et al., 2006). A difference in transcript levels between strains $\mathrm{T} 9$ and $\mathrm{S} 3$ by up to a factor 10 was shown in Fig. 1B and C. An earlier study using Northern hybridization of plc mRNA had also reported large variations in transcript levels between strains, up to 40-fold (Tsutsui et al., 1995). Surprisingly, strain T9, with the lower transcript levels, produced the higher amount of $\alpha$-toxin, i.e., $0.49 \mathrm{U} \mathrm{ml}^{-1}$ in late stationary phase vs. $0.13 \mathrm{U} \mathrm{ml}^{-1}$ produced by the highly plc-transcribing strain S3 (Fig. 1D). This is in contrast to earlier findings of high correlation between plc mRNA and $\alpha$-toxin levels also in strains showing different levels of phospholipase C activity (Bullifent et al., 1996). The plc gene of strain S3 contains an 834 bp-intron (Abildgaard et al., 2009), previously found also in other $C$. perfringens strains (Ma et al., 2007), which theoretically could decrease translation efficiencies due to the need for mRNA processing (splicing). However, no un-spliced mRNA has been detected in these strains (Abildgaard et al., 2009; Ma et al., 2007), indicating rapid mRNA processing. The most probable explanation for the discrepancy in apparent transcription and translation levels between strains T9 and S3 is therefore that both features are strain-specific.

\subsection{Implications for monitoring the effect of NE treatment}

Transcript levels of toxin genes have been used to monitor the efficiency of treatments against Salmonella (Lim et al., 2007), Vibrio (Lim et al., 2007), and C. botulinum (Sharkey et al., 2005; Shin et al., 2006). Likewise, plc gene copy numbers and transcript levels may be used to evaluate the effect of treatments on number and $\alpha$-toxin production of $C$. perfringens in pure cultures of known strain types. Towards that end, our revised qPCR probe/ primer set offers a reliable tool to determine $\alpha$-toxin gene copy numbers and, combined with RT and normalization against gyrA or rplL mRNA, $\alpha$-toxin gene transcript levels. However, due to the putative induction of plc transcription during exponential growth, different strains need to be in the same growth phase when comparing treatment effects on plc transcript levels. Finally, it is crucial to note that according to our findings, plc gene transcript (mRNA) levels can only be used to predict the amount of $\alpha$-toxin produced when knowing the degree of translational regulation for the strains in question. The protocol can be further developed to measure the effect on $\mathrm{NE}$ treatments on plc transcription and $\alpha$-toxin production in intestinal content of broilers during challenge trials. Again for this purpose the relationship between plc transcription and $\alpha$-toxin production of the strain(s) used for infection should be assessed in vitro.

\section{Acknowledgements}

The study was supported by the EC-funded FP6 project REPLACE (Food-CT-2004-506487). The authors wish to thank K. Pedersen (Nauerby et al., 2003) for supplying the strains.

\section{Appendix A. Supplementary data}

Supplementary data associated with this article can be found, in the online version, at doi:10.1016/j.vetmic. 2009.05.014. 


\section{References}

Abildgaard, L., Engberg, R.M., Pedersen, K., Schramm, A., Højberg, O., 2009. Sequence variation in the $\alpha$-toxin encoding plc gene of Clostridium perfringens strains isolated from diseased and healthy chickens. Vet. Microbiol. 136, 293-299.

Alifano, P., Bruni, C., Carlomagno, M., 1994. Control of mRNA processing and decay in prokaryotes. Genetica 94, 157-172.

Al-Sheikhly, F., Truscott, R.B., 1977a. The pathology of necrotic enteritis of chickens following infusion of crude toxins of Clostridium perfringens into the duodenum. Avian Dis. 21, 241-255.

Al-Sheikhly, F., Truscott, R.B., 1977b. The pathology of necrotic enteritis of chickens following infusion of broth cultures of Clostridium perfringens into the duodenum. Avian Dis. 21, 230-240.

Awad, M.M., Bryant, A.E., Stevens, D.L., Rood, J.I., 1995. Virulence studies on chromosomal alpha-toxin and theta-toxin mutants constructed by allelic exchange provide genetic evidence for the essential role of alpha toxin in Clostridium perfringens mediated gas gangrene. Mol. Microbiol. 15, 191-202.

Bullifent, H.L., Moir, A., Awad, M.M., Scott, P.T., Rood, J.I., Titball, R.W., 1996. The level of expression of alpha-toxin by different strains of Clostridium perfringens is dependent on differences in promoter structure and genetic background. Anaerobe 2, 365-371.

Cooper, K.K., Trinh, J., Songer, J.G., 2009. Immunization with recombinant alpha toxin partially protects broiler chicks against challenge with Clostridium perfringens. Vet. Microbiol. 133, 92-97.

Cowen, B.S., Schwartz, L.D., Wilson, R.A., Ambrus, S.I., 1987. Experimentally induced necrotic enteritis in chickens. Avian Dis. 31, 904-906.

Engberg, R.M., Hedemann, M.S., Jensen, B.B., 2002. The influence of grinding and pelleting of feed on the microbial composition and activity in the digestic tract of broiler chickens. Brit. Poult. Sci. 44 , 569-579.

Fernandez-Miyakawa, M.E., Marcellino, R., Uzal, F.A., 2007. Clostridium perfringens type A toxin production in 3 commonly used culture media. J. Vet. Diagn. Invest. 19, 184-186.

Ficken, M.D., Wages, D.P., 1997. Necrotic enteritis. In: Calnek, B.W. (Ed.), Diseases of Poultry. Iowa State University Press, pp. 261-264.

Fukata, T., Hadate, Y., Baba, E., Arakawa, A., 1991. Influence of bacteria on Clostridium perfringens infections in young chickens. Avian Dis. 35, 224-227.

Fukata, T., Hadate, Y., Baba, E., Uemura, T., Arakawa, A., 1988. Influence of Clostridium perfringens and its toxin in germ-free chickens. Res. Vet. Sci. $44,68-70$.

Gholamiandekhordi, A.R., Ducatelle, R., Heyndrickx, M., Haesebrouck, F., Van Immerseel, F., 2006. Molecular and phenotypical characterization of Clostridium perfringens isolates from poultry flocks with different disease status. Vet. Microbiol. 113, 143-152.

Huang, W.M., 1996. Bacterial diversity based on type II DNA topoisomerase genes. Annu. Rev. Genet. 30, 79-107.

Keyburn, A.L., Boyce, J., Vaz, P., Bannam, T.L., Ford, M.E., Parker, D., Di Rubbo, A., Rood, J.I., Moore, R.J., 2008. NetB, a new toxin that is associated with avian necrotic enteritis caused by Clostridium perfringens. PLOS Pathogens 4, e26.

Kulkarni, R.R., Parreira, V.R., Sharif, S., Prescott, J.F., 2007. Immunization of broiler chickens against Clostridium perfringens-induced necrotic enteritis. Clin. Vaccine Immunol. 14, 1070-1077.

Kulkarni, R.R., Parreira, V.R., Sharif, S., Prescott, J.F., 2008. Oral immunization of broiler chickens against necrotic enteritis with an attenuated Salmonella vaccine vector expressing Clostridium perfringens antigens. Vaccine 26, 4194-4203.

Kutyavin, I.V., Afonina, I.A., Mills, A., Gorn, V.V., Lukhtanov, E.A., Belousov, E.S., Singer, M.J., Walburger, D.K., Lokhov, S.G., Gall, A.A., Dempcy, R., Reed, M.W., Meyer, R.B., Hedgpeth, J., 2000. 3'-Minor groove binder-
DNA probes increase sequence specificity at PCR extension temperatures. Nucleic Acid Res. 28, 655-661.

Lim, S., Jung, J., Kim, D., 2007. The effect of $\gamma$ radiation on the expression of the virulence genes of Salmonella typhimurium and Vibrio spp. Radiat. Phys. Chem. 76, 1763-1766.

Ma, M., Ohtani, T., Misawa, N., 2007. Detection of a group II intron without an open reading frame in the alpha-toxin gene of Clostridium perfringens isolated from a broiler chicken. J. Bacteriol. 189, 1633-1640.

Möllby, R., Holme, T., Nord, C.E., Smyth, C.J., Wadstrom, T., 1976. Production of phospholipase C (alpha-toxin), haemolysins and lethal toxins by Clostridium perfringens types A to D. J. Gen. Microbiol. 96, 137-144.

Nauerby, B., Pedersen, K., Madsen, M., 2003. Analysis by pulsed-field gel electrophoresis of the genetic diversity among Clostridium perfringens isolates from chickens. Vet. Microbiol. 94, 257-266.

Olkowski, A.A., Wojnarowicz, C., Chirino-Trejo, M., Laarveld, B., Sawicki, G., 2008. Sub-clinical necrotic enteritis in broiler chickens: novel etiological consideration based on ultra-structural and molecular changes in the intestinal tissue. Res. Vet. Sci. 85, 543-553.

Pedersen, K., Bjerrum, L., Nauerby, B., Madsen, M., 2003. Experimental infections with rifampicin-resistant Clostridium perfringens strains in broiler chickens using isolator facilities. Avian Pathol. 32, 403-411.

Ramakrishnan, V., Moore, P.B., 2001. Atomic structures at last: the ribosome in 2000. Curr. Opin. Struct. Biol. 11, 144-154.

Rooney, A.P., Swezey, J.L., Friedman, R., Hecht, D.W., Maddox, C.W., 2006. Analysis of core housekeeping and virulence genes reveals cryptic lineages of Clostridium perfringens that are associated with distinct disease presentations. Genetics 172, 2081-2092.

Rudi, K., Nogva, H.K., Naterstad, K., Drømtorp, S.M., Bredholt, S., Holck, A., 2003. Subtyping Listeria monocytogenes through the combined analyses of genotype and expression of the hlyA virulence determinant. J Appl. Microbiol. 94, 720-732.

Sharkey, F.H., Dooley, J.S., Haylock, R.W., 2005. Quantitative effects of carbohydrates and aromatic amino acids on Clostridium botulinum toxin gene expression using a rapid competitive RT/PCR assay. J. Mol. Microbiol. Biotechnol. 9, 35-43.

Shimizu, T., Ohtani, K., Hirakawa, H., Ohshima, K., Yamashita, A., Shiba, T., Ogasawara, N., Hattori, M., Kuhara, S., Hayashi, H., 2002. Complete genome sequence of Clostridium perfringens, an anaerobic flesh-eater. Proc. Natl. Acad. Sci. U.S.A. 99, 996-1001.

Shin, N.-R., Shin, J.-H., Chun, J.H., Yoon, S.-Y., Kim, B.S., Oh, H.-B., Rhie, G.E., 2006. Determination of neurotoxin gene expression in Clostridium botulinum Type A by quantitative RT-PCR. Mol. Cells 22, 336-342.

Skånseng, B., Kaldhusdal, M., Rudi, K., 2006. Comparison of chicken gut colonisation by the pathogens Campylobacter jejuni and Clostridium perfringens by real-time quantitative PCR. Mol. Cell Probe 20, 269279.

Songer, J.G., 1996. Clostridial enteric diseases of domestic animals. Clin. Microbiol. Rev. 9, 216-234.

Thompson, D.R., Parreira, V.R., Kulkarni, R.R., Prescott, J.F., 2006. Live attenuated vaccine-based control of necrotic enteritis of broiler chickens. Vet. Microbiol. 113, 25-34.

Tsutsui, K., Minami, J., Matsushita, O., Katayama, S., Taniguchi, Y., Nakamura, S., Nishioka, M., Okabe, A., 1995. Phylogenetic analysis of phospholipase C genes from Clostridium perfringens types A to E and Clostridium novyi. J. Bacteriol. 177, 7164-7170.

Werbrouck, H., Botteldoorn, N., Uyttendaele, M., Herman, L., Van Coillie, E., 2007. Quantification of gene expression of Listeria monocytogenes by real-time reverse transcription PCR: optimization, evaluation and pitfalls. J. Microbiol. Methods 69, 306-314.

Williamson, E.D., Titball, R.W., 1993. A genetically engineered vaccine against the alpha-toxin of Clostridium perfringens also protects mice against experimental gas gangrene. Vaccine 11, 1253-1258. 